\title{
Exclusion of the dymeclin and PAPSS2 genes in a novel form of spondyloepimetaphyseal dysplasia and mental retardation
}

David Geneviève ${ }^{1}$, Delphine Héron ${ }^{2}$, Vincent El Ghouzzi ${ }^{1}$, Catherine Prost-Squarcioni ${ }^{3}$, Martine Le Merrer ${ }^{1}$, Aurélia Jacquette ${ }^{2}$, Damien Sanlaville ${ }^{1}$, Florence Pinton ${ }^{4}$, Nathalie Villeneuve $^{4}$, Gabriel Kalifa ${ }^{5}$, Arnold Munnich ${ }^{1}$ and Valérie Cormier-Daire*,1

${ }^{1}$ Département de Génétique et Unité INSERM U393, Hôpital Necker-Enfants Malades, Paris, France; ${ }^{2}$ Département de Génétique, Hôpital de la Pitié-Salpêtrière, Paris, France; ${ }^{3}$ CNRS UPRES 3410, Faculté de Médecine, Hôpital Avicenne, Bobigny, France; ${ }^{4}$ Service de Neuropédiatrie, Hôpital Saint Vincent de Paul, Paris, France; ${ }^{5}$ Service de Radiologie, Hôpital Saint Vincent de Paul, Paris, France

Spondyloepimetaphyseal dysplasias (SEMD) represent a heterogeneous group of conditions composed of at least 15 well-defined entities. The classification is based on clinical, radiological and molecular findings. Among them, several conditions also include a mental retardation (MR) syndrome, namely Wolcott-Rallison syndrome, Dyggve-Melchior-Clausen syndrome (DMC) and lysosomal storage disorders. Here, we report on a novel form of SEMD with MR in two Pakistani sisters born to first-cousin parents. SEMD, MR, microcephaly, ataxia, facial dysmorphism and hirsutism of back and legs were noted in the two children. Skeletal findings included flat vertebral bodies with irregular vertebral plates, irregular and flared metaphyses with vertical striations, small and irregular epiphyses, small carpal bones and narrow iliac wings without lacy pelvis iliac crest. Similarities with DMC prompted us to test and eventually exclude the DMC gene, dymeclin, by direct sequencing. Similarly, we excluded the PAPSS2 gene ( $3^{\prime}$-alpha phosphoadenosine $5^{\prime}$-phosphosulphate synthase 2 ) responsible for SEMD Pakistani type. The combination of features observed in the two sisters does not fit with any previously reported SEMD and represents therefore a novel form of autosomal recessive SEMD with MR.

European Journal of Human Genetics (2005) 13, 541-546. doi:10.1038/sj.ejhg.5201339

Published online 23 February 2005

Keywords: spondyloepimetaphyseal dysplasia; mental retardation; autosomal recessive

\section{Introduction}

Spondyloepimetaphyseal dysplasias (SEMD) represent a heterogeneous group of conditions composed of at least

*Correspondence: $\operatorname{Dr} \mathrm{V}$ Cormier-Daire, Département de Génétique, Hôpital Necker-Enfants Malades, 149, rue de Sèvres, 75743 Paris Cedex, France. Tel: + 3314449 5163; Fax: + 3314449 5150;

E-mail: cormier@necker.fr

Received 29 June 2004; revised 21 September 2004; accepted 23 September 2004
15 well-defined entities. These various forms of SEMD have been characterised based on clinical, radiological and molecular features, but only few of them are associated with mental retardation (MR), ${ }^{1}$ namely (i) WolcottRallison syndrome ${ }^{2-3}$ (WR, MIM 226980) due to mutations in the EIF2AK3 gene encoding a translation initiation factor 2-alpha kinase 3 and regulating the synthesis of unfolded proteins in the endoplasmic reticulum, ${ }^{4}$ (ii) Dyggve-Melchior-Clausen syndrome ${ }^{5}$ (DMC, MIM 223800) due to mutations in the dymeclin gene encoding 
a protein of hitherto unknown function and (iii) lysosomal storage disorders. ${ }^{6}$ Here, we report on what we believe to be a novel autosomal recessive syndrome of SEMD and MR in two Pakistani sisters.

\section{Patients report}

Patient 1 was a girl born to first-cousin Pakistani parents after term pregnancy and normal delivery (birth weight $3 \mathrm{~kg}$, height and OFC were unknown). Hypotonia, nystagmus with poor vision and feeding difficulties were noted in the neonatal period. She was able to control her head at 11 months and to sit unaided at 2 years but her gait was unstable. At $8 \frac{1}{2}$ years, she had no speech, had ataxia and was unable to walk unaided (weight $=18.8 \mathrm{~kg} ;-2 \mathrm{SD}$; height $=104 \mathrm{~cm}, \quad-4.5 \mathrm{SD} ; \quad \mathrm{OFC}=46.5 \mathrm{~cm}, \quad-5 \mathrm{SD})$. No abnormal body proportion was observed. Facial dysmorphism included coarse facies, low anterior and posterior hairline, brachycephaly, simple and flat ears, small and well-shaped eyebrows, synophrys, bilateral epicanthi, broad nose, full lips with eversion of the lower lip, short neck and hirsutism of back and legs (Figure 1a). At the last examination (11 years), she could walk unaided but with ataxia and her coarse facial appearance had worsened.

Patient 2, her younger sister, was born after an uneventful pregnancy (birth weight $=2500 \mathrm{~g}$, length and OFC were unknown). She could sit at 2 years of age and walk unaided
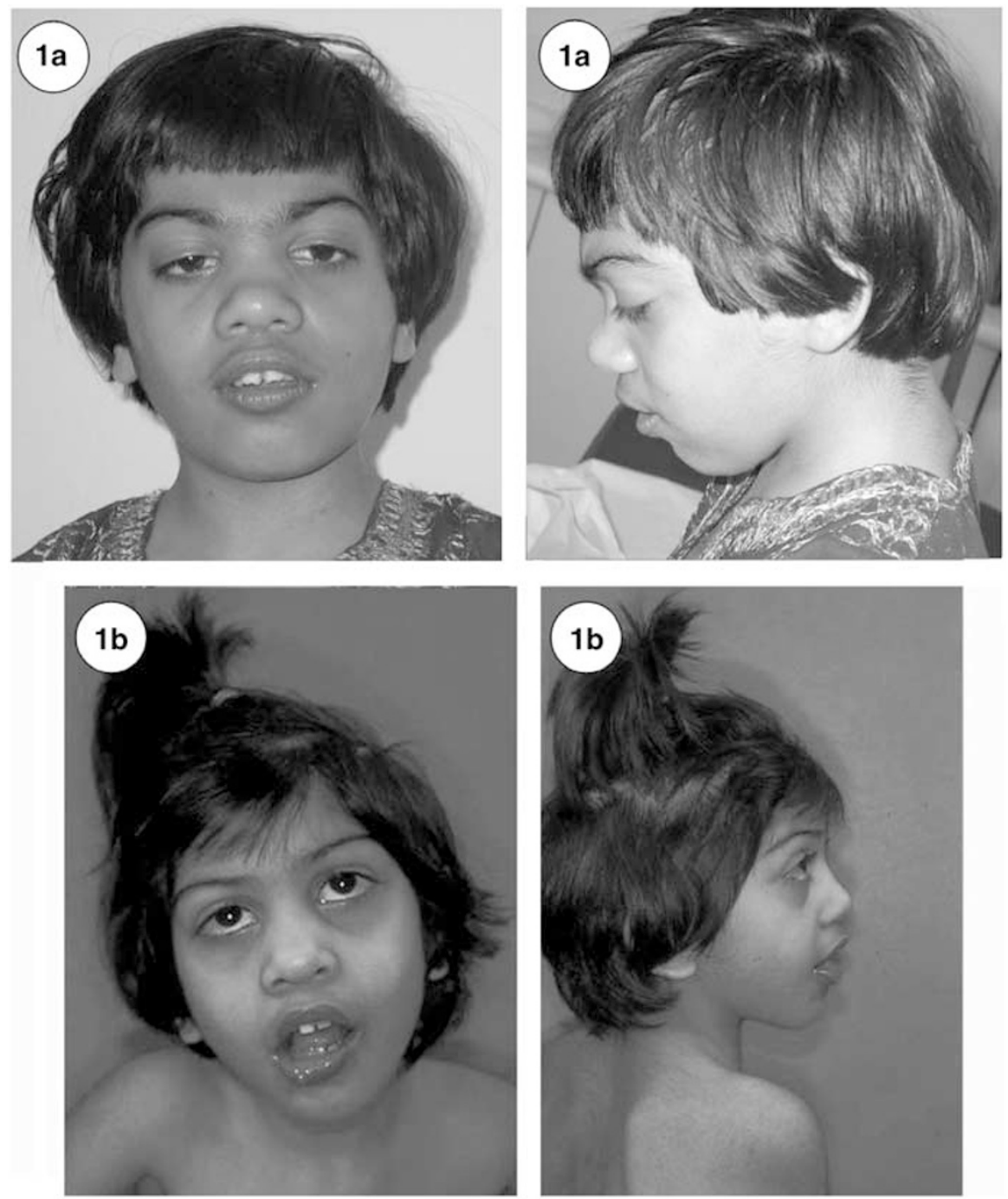

Figure 1 (a) Front and lateral view of patient 1 at 11 years of age. Note facial dysmorphism including coarse facies, low anterior and posterior hairline, brachycephaly, simple and flat ears, small and well-shaped eyebrows, synophrys, bilateral epicanthi, broad nose, full lips with eversion of the lower lip, short neck and hirsutism on back and legs. (b) Front and lateral view of patient 2 at 7 years of age. Facial dysmorphism is similar but less coarse than that of the older sister. 

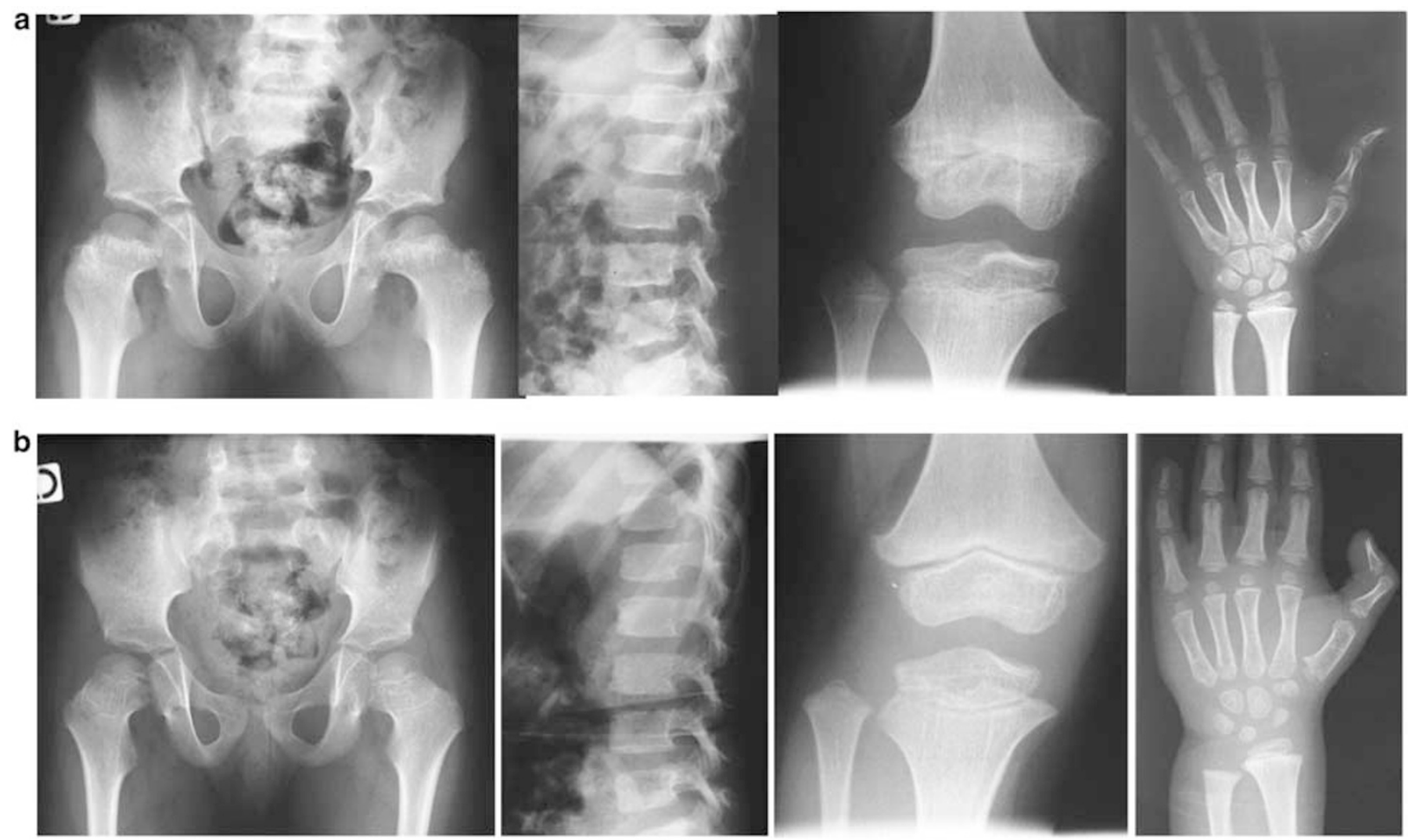

Figure 2 (a) Skeletal anomalies observed in patient 1 at 10 years of age. Note narrow iliac wings without lacy pelvis iliac crest, flat vertebral bodies with irregular vertebral plates and posterior scalloping body, irregular and flared metaphyses with vertical striations, small and irregular epiphysis, small carpal bones. Note also flat acetabular roofs, short femoral neck and disproportionately long fibulae. (b) Bone X-rays of patient 2 at 6 years of age showed less severe but quite similar bone involvement than observed in her older sister.

at $3 \frac{1}{2}$ years. She was first seen at 32 months of age for hypotonia with trunk and gait ataxia (weight $=9.8 \mathrm{~kg},-2.5$ $\mathrm{SD}$; length $=83.5 \mathrm{~cm},-2 \mathrm{SD} ; \mathrm{OFC}=43 \mathrm{~cm},-5 \mathrm{SD})$. She also had repetitive behaviour and no speech. Facial features included brachycephaly, low anterior and posterior hairline, bilateral epicanthus, simple and flat ears with everted lobules, broad nose and full lips with eversion of the lower lip. She also had abnormal pigmentary skin changes on the legs along the Blaschkoid lines. No abnormal body proportion was observed. At 5 years of age, her coarse facial appearance and failure to thrive had worsened (weight $=11 \mathrm{~kg}, \quad-3.5 \mathrm{SD}$; length $=90 \mathrm{~cm}, \quad-4 \mathrm{SD}$; $\mathrm{OFC}=45 \mathrm{~cm},-5 \mathrm{SD}$; Figure $1 \mathrm{~b}$ ).

\section{Laboratory investigations}

Heart and kidney ultrasounds, brain MRI, fundus examination, evoked visual potential, electromyogram, standard and high-resolution blood karyotype and FISH analyses of the telomeres were normal on the two sibs. Plasma and urinary amino acids and organic acids, plasma T3, T4, TSH, protein glycosylation tests (Western blot analysis) and blood and fibroblast lysosomal enzymes ( $\beta$-glucosidase, $\beta$ galactosidase, hexosaminidase A and B, $\alpha$-L-iduronidase, $\alpha$ - glucosaminidase, $\alpha$-galactose 6 sulphatase, arylsulphatase A and $\mathrm{B}, \alpha$ - and $\beta$-mannosidase, $\alpha$-fucosidase, $\alpha$-neuraminidase and $\alpha$ - $\underline{N}$-acetylgalactosaminidase) were unremarkable. Molecular analysis of the MECP2 gene was also normal. Electroretinogram of patient 1 detected an abnormal macular signal but normal peripheral retina.

\section{Methods \\ Molecular studies}

DNA was extracted from peripheral blood lymphocytes according to standard techniques. ${ }^{7}$ Because of noninformative haplotype analysis of the DMC locus, direct sequencing of the DMC gene, dymeclin, was performed using primers previously described. ${ }^{8}$ An intragenic polymorphic microsatellite marker was used to analyse the PAPSS2 (3'-alpha phosphoadenosine 5'-phosphosulphate synthase 2) gene, which accounts for the SEMD Pakistani type (PAPSS2 5' GGC AGT AAG TTT GCA GTA GA; PAPSS2 3' GTC TAC AAC CCA AGT CT).

\section{Electron microscopy and ultrastructural analyses}

A $4 \mathrm{~mm}$ punch skin biopsy including dermis was performed in both sisters. The sample was fixed in $2.5 \%$ glutaralde- 


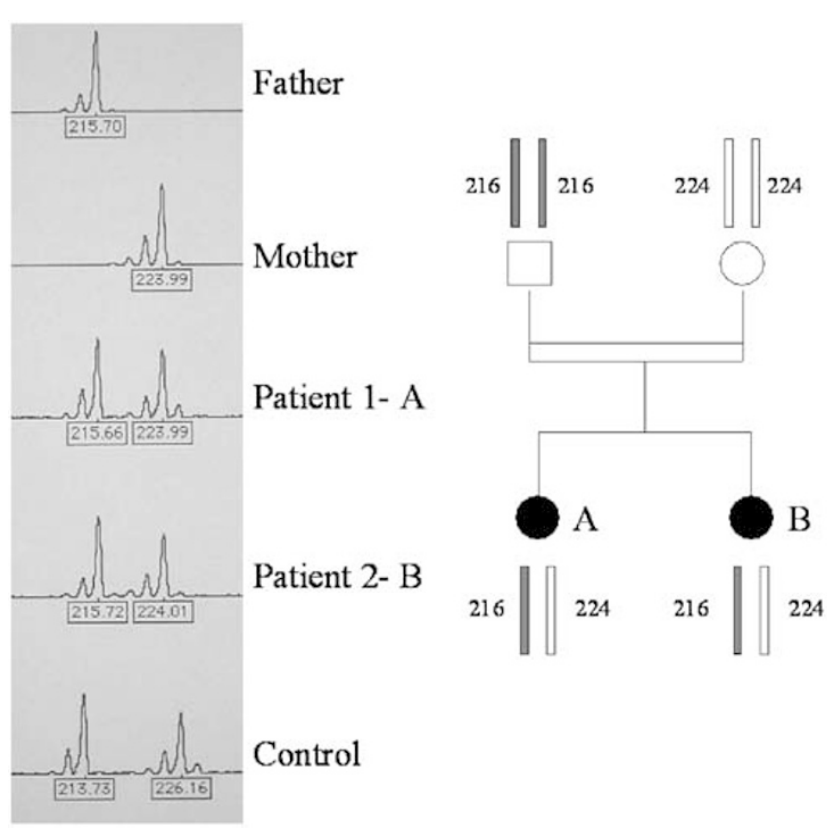

Figure 3 Electrophoregram and segregation of the PAPSS2 intragenic polymorphic microsatellite marker in our family.

hyde, postfixed in 1\% osmium tetroxide, dehydrated in a graded ethanol series and embedded in epon 812 . Semithin sections $(1 \mu \mathrm{m})$ were stained with toluidine blue. Ultrathin sections were selected, contrasted with uranyl acetate and lead citrate and examined with a Philips CM 10 transmission electron microscope.

\section{Results}

Skeletal X-rays of patient 1 showed radial incurvation and SEMD including flat vertebral bodies with irregular vertebral plates and posterior scalloping, irregular and flared femoral superior and inferior, radial and ulnar as well as fibulae and tibial superior metaphyses with vertical striations, small and irregular femoral superior and inferior as well as tibial superior epiphyses, small carpal bones, flat acetabular roofs, short femoral neck, disproportionate long fibulae and narrow iliac wings without lacy pelvis iliac crest (Figure 2a).

In patient 2, skeletal X-rays showed similar, but less severe, bone involvement including radial incurvation and SEMD with flat vertebral bodies and posterior scalloping, irregular vertebral plates, irregular and flared femoral superior and inferior, radial and ulnar as well as fibulae and tibial superior metaphyses with vertical striations, small and irregular femoral superior and inferior as well as tibial superior epiphyses with small carpal bones, flat acetabular roofs, short femoral neck, disproportionate long fibulae and narrow iliac wings without lacy pelvis iliac crest (Figure 2b).
Molecular studies allow us to exclude the dymeclin gene by direct sequencing and the PAPSS2 gene by linkage analysis (Figure 3).

Electron microscopy analysis of the skin biopsy of affected children revealed an abnormally large number of small vacuoles near the cytoplasmic membrane in fibroblasts, macrophages (Figure $4 \mathrm{a}-\mathrm{c}$ ) and cells of the duct canal portion of the sweat glands (Figure $4 \mathrm{~d}$ and e). Moreover, large vacuoles containing either myelinic or granular osmiophilic bodies were observed in most cutaneous cell types studied, particularly in fibroblasts, macrophages, keratinocytes, melanocytes, secretory and duct canal portions of the sweat glands. Other features included dilated endoplasmic reticulum in fibroblasts, while Golgi apparatus and mitochondria were normal.

\section{Discussion}

We report here on SEMD, severe MR, microcephaly, facial dysmorphism and ataxia in two Pakistani sisters born to first-cousin parents. Severity of the clinical and radiological manifestations worsened in the course of the disease in the two sibs. The association of SEMD and MR prompted us to consider the diagnosis of $\mathrm{DMC},{ }^{8-10}$ but molecular genetic studies and distinct radiological features (ie lacy pelvis iliac crest and double vertebral humps) ruled out this diagnosis. Similarly, the absence of insulin-dependent diabetes mellitus and distinctive radiological features ruled out the diagnosis of WR syndrome as well.

The radiological features were reminiscent of the SEMD Pakistani type (especially vertebral anomalies and epiphyseal dysplasia), ${ }^{11}$ and the combination of these findings with the ethnic origin of the family and the likely autosomal recessive mode of inheritance prompted us to study the PAPSS2 locus. ${ }^{12}$ However, the MR observed in our patients and the coarse facial appearance that worsened in the course of the disease have never been reported in SEMD Pakistani type. In addition, the molecular study using an intragenic polymorphic marker allows us to exclude the PAPSS2 gene as the disease locus gene.

Metaphyseal striations are a rare radiological feature observed in several skeletal conditions, but only a few of these are associated with MR. Among them, SPONASTRIME dysplasia (MIM: 271510) is characterised by Spondylo (ie platyspondyly and irregular vertebral end-plates), nasal anomalies, striations of metaphyses and epiphyseal dysplasia. MR has been described in two families ${ }^{13,14}$ but the absence of vertebral body abnormalities has suggested that these patients have a different condition. ${ }^{15}$ Moreover, the combination of dysmorphic and radiological features, observed in SPONASTRIME dysplasia or related conditions, is clearly distinct from the new SEMD form reported here. 

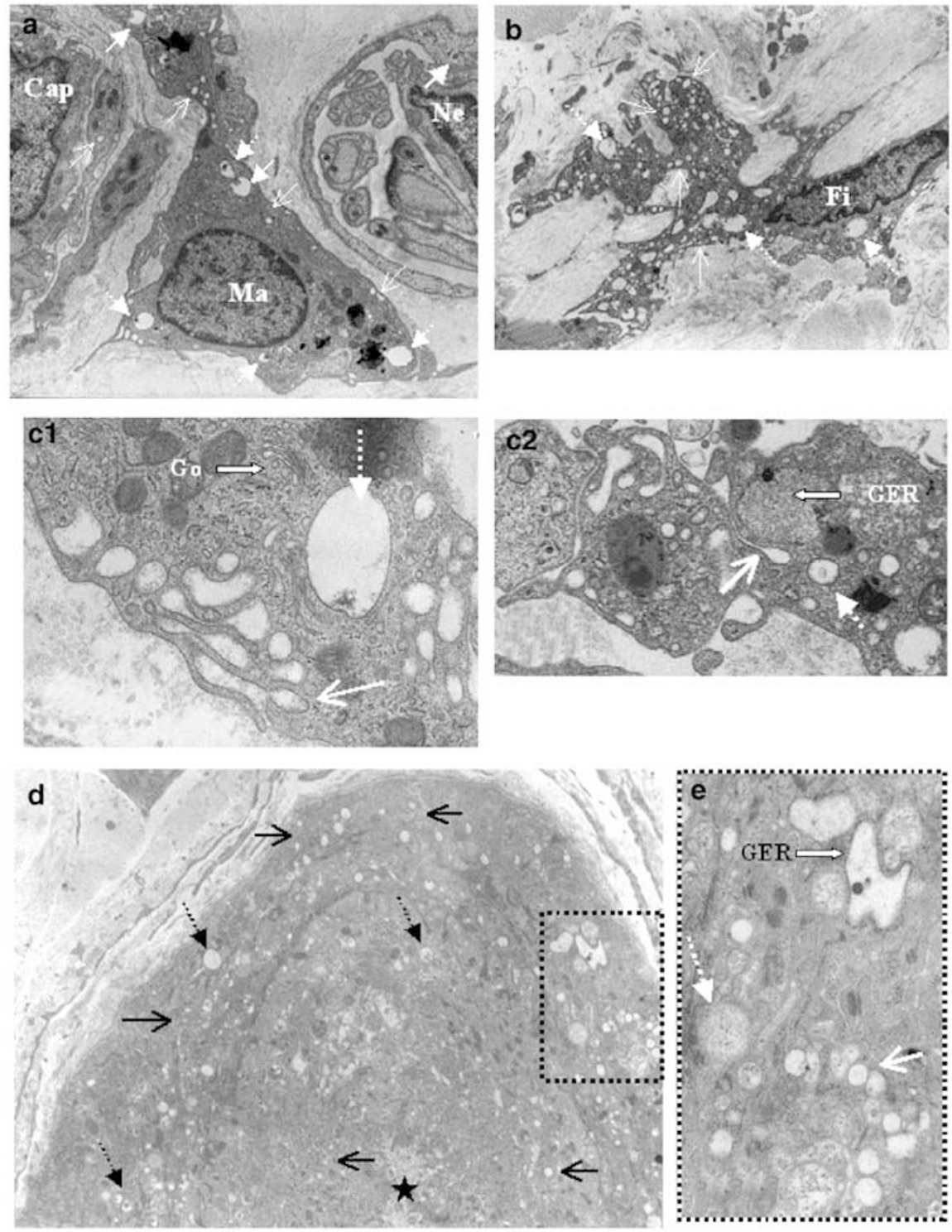

Figure $4(\mathbf{a}-\mathbf{c})$ Electron microscopy analysis of the skin biopsy of patient 1: cytoplasm of a macrophage (a) and a fibroblast (b) display abnormally numerous phagocytosis vacuoles $(\rightarrow)$ along the cytoplasmic membrane and large vacuoles, which contain either myelinic bodies $(\rightarrow$ ) or granular osmiophilic bodies $(\cdot \rightarrow)$. The fusion of the phagocytosis vacuoles with the cytoplasmic membrane $(\rightarrow)(C 1-C 2)$, dilated GER (C2) and normal Golgi apparatus (C1) in two other fibroblasts is shown. Ma: nucleus of a macrophage; Ne: nucleus of a Schwann cell in a small nerve; Cap: nucleus of an endothelial cell in a capillary; Fi: nucleus of a fibroblast; GER: granulous endoplasmic reticulum; Go: Golgi apparatus. (d, e) Electron microscopy analysis of the skin biopsy of patient 2: cells of the two layers of the duct canal of sweat glands contain numerous small vacuoles $(\rightarrow$ ) usually along the cytoplasmic membrane and larger vacuoles, which contain granular osmiophilic bodies (..-). D: low magnification; E: higher magnification of the inset; GER: dilated granulous endoplasmic reticulum; is: lumen of the duct canal of a sweat gland.

Despite negativity of extensive blood and urinary metabolic screening, the progressivity of organ involvement and electron microscopy studies of the skin (abnormally large number of small vacuoles in fibroblasts, macrophages, keratinocytes, melanocytes, secretory and duct canal portion of sweat glands) observed in our patients may argue in favour of a novel hitherto unknown metabolic disorder.
We suggest therefore that the combination of features observed in this sibship corresponds to a novel form of autosomal recessive SEMD with MR, especially as severity of symptoms worsened in the course of the disease.

\section{Acknowledgements}

We thank Jean-Paul Monnet and Nicole Lièvre for their technical assistance for electron microscopy. Financial support from the 
association 'Vaincre les maladies lysosomales' is gratefully acknowledged.

\section{References}

1 Hall CM: International nosology and classification of constitutional disorders of bone (2001). Am J Med Genet 2002; 113: 65-77.

2 Wolcott CD, Rallison ML: Infancy-onset diabetes mellitus and multiple epiphyseal dysplasia. J Pediatr 1972; 80: 292-297.

3 Delepine M, Nicolino M, Barrett T, Golamaully M, Lathrop GM, Julier C: EIF2AK3, encoding translation initiation factor 2-alpha kinase 3 , is mutated in patients with Wolcott-Rallison syndrome. Nat Genet 2000; 25: 406-409.

4 Zhang P, McGrath B, Li S et al: The PERK eukaryotic initiation factor 2 alpha kinase is required for the development of the skeletal system, postnatal growth, and the function and viability of the pancreas. Mol Cell Biol 2002; 22: 3864-3874.

5 Dyggve HV, Melchior JC, Clausen J: Morquio-Ullrich's disease: an inborn error of metabolism? Arch Dis Child 1962; 37: $525-534$

6 Maroteaux P, Le Merrer M: Maladies osseuses de l'enfant. Flammarion, Paris: Medecine Science, 2003, 4th edn.

7 Sambrook J, Fritsch EF, Maniatis T: Molecular Cloning: A Laboratory Manual. Cold Spring Harbor Laboratory Press: Cold Spring Harbor, NY, 1989, 2nd edn.
8 El Ghouzzi V, Dagoneau N, Kinning E et al: Mutations in a novel gene Dymeclin (FLJ20071) are responsible for Dyggve-MelchiorClausen syndrome. Hum Mol Genet 2003; 12: 357-364.

9 Thauvin-Robinet C, El Ghouzzi V, Chemaitilly W et al: Homozygosity mapping of a Dyggve-Melchior-Clausen syndrome gene to chromosome 18q21.1. J Med Genet 2002; 39: 714-717.

10 Ehtesham N, Cantor RM, King LM et al: Evidence that SmithMcCort dysplasia and Dyggve-Melchior-Clausen dysplasia are allelic disorders that result from mutations in a gene on chromosome 18q12. Am J Hum Genet 2002; 71: 947-951.

11 Ahmad M, Haque MF, Ahmad W et al: Distinct, autosomal recessive form of spondyloepimetaphyseal dysplasia segregating in an inbred Pakistani kindred. Am J Med Genet 1998; 78: 468473.

12 ul Haque MF, King LM, Krakow D et al: Mutations in orthologous genes in human spondyloepimetaphyseal dysplasia and the brachymorphic mouse. Nat Genet 1998; 20: 157-162.

13 Camera G, Camera A, Di Rocco M, Gatti R: Sponastrime dysplasia: report on two siblings with metal retardation. Pediatr Radiol 1993; 23: $611-614$.

14 Verloes A, Misson JP, Dubru JM, Jamblin P, Le Merrer M: Heterogeneity of SPONASTRIME dysplasia: delineation of a variant form with severe mental retardation. Clin Dysmorphol 1995; 4: 208-215.

15 Langer Jr LO, Beals RK, LaFranchi S, Scott Jr CI, Sockalosky JJ: Sponastrime dysplasia: five new cases and review of nine previously published cases. Am J Med Genet 1996; 63: 20-27. 